\title{
Comparison of Three Instruments for Activity Disability in Acute Ischemic Stroke Survivors
}

\author{
Qian Wu (D), Aijie Tang, Shuzhen Niu, Aiping Jin, Xiaoqing Liu, Li Zeng, \\ Jinxia Jiang, Jennifer Kue, Yan Shi, Xiaoping Zhu
}

\begin{abstract}
Background: Disabilities in physical activity and functional independence affect the early rehabilitation of stroke survivors. Moreover, a good instrument for assessing activity disability allows accurate assessment of physical disability and assists in prognosis determination. Objective: To compare three assessment tools for physical activity in acute-phase stroke survivors. Methods: We conducted this prospective observational study at an affiliated hospital of a Medical University in Shanghai, China, from June 2018 to November 2019. We administered three instruments to all patients during post-stroke days 5-7, including the Modified Barthel Index (MBI), Instrumental Activities of Daily Living (IADL), and modified Rankin scale (mRs). We analyzed correlations among the aforementioned scales and the National Institutes of Health Stroke Scale (NIHSS) using Spearman's rank-order correlations test. Univariate analyses were performed using the Mann-Whitney U test. We used a binary logistic regression model to assess the association between the NIHSS (30 days) and patient-related variables. Finally, we used receiver operating characteristic (ROC) curves to assess the predictive value of the multivariate regression models. Results: There was a high correlation among the three instruments; furthermore, the MBI had a higher correlation with the NIHSS (days 5-7). The NIHSS (day 30) was correlated with thrombolysis. ROC analysis revealed that the mRs-measured disability level had the highest predictive value of short-term stroke severity (30 days). Conclusion: The MBI was the best scale for measuring disability in physical activity, whereas the mRs showed better accuracy in short-term prediction of stroke severity.
\end{abstract}

RÉSUMÉ : Comparaison entre trois outils d'évaluation de l'invalidité dans le cas de patients ayant survécu à un AVC ischémique aigu. Contexte : Des limitations fonctionnelles en matière d'activité physique et d'autonomie vont affecter les premières étapes de la réadaptation des survivants à un AVC. Par ailleurs, on sait qu'un bon outil d'évaluation de ces limitations permettra de les évaluer adéquatement et d'établir un pronostic. Objectif : Comparer trois outils d'évaluation des limitations fonctionnelles liées à l'activité physique dans le cas de survivants à un AVC. Méthodes: De juin 2018 à novembre 2019, nous avons effectué une étude prospective d'observation au sein d'un établissement hospitalier affilié à une école de médecine de Shanghai. Entre les cinquièmes et septièmes jours consécutifs à un AVC, tous nos patients ont été évalués au moyen des trois outils suivants : l'indice modifié de Barthel (IMB), l'échelle de Lawton ciblant les activités instrumentales de la vie quotidienne et la Modified Rankin Scale (MRS). Au moyen du test de Spearman, nous avons ensuite analysé les corrélations se dessinant entre ces trois outils et les résultats obtenus avec un autre outil, la National Institutes of Health Stroke Scale (NIHSS). Nos analyses univariées ont été par ailleurs effectuées à l'aide du test de Wilcoxon-Mann-Whitney. Nous avons également recouru à un modèle de régression logistique binaire afin d'évaluer l'association existant entre le NIHSS (30 jours) et des variables liées aux patients. Finalement, nous avons fait appel à la fonction d'efficacité du récepteur (ou courbe ROC) pour évaluer la valeur prédictive de nos modèles de régression multivariés. Résultats : Une forte corrélation entre nos trois outils a émergé. De plus, il est apparu que c'est le IMB qui a montré la plus forte corrélation avec la NIHSS (jours 5, 6 et 7). La NIHSS (jour 30) a été également corrélée avec un traitement thrombolytique. Nos analyses au moyen de la fonction d'efficacité du récepteur ont révélé que le niveau de limitations fonctionnelles mesuré par la MRS avait la valeur prédictive la plus élevée pour le degré de sévérité des AVC à court terme (jour 30). Conclusion : L'IMB est donc apparu comme le meilleur outil pour évaluer les limitations fonctionnelles en matière d'activité physique tandis que la MRS a montré la meilleure précision quand il s'agit de prédire à court terme le niveau de sévérité des AVC.

Keywords: Stroke, Activity, Disability, Modified Rankin scale, Modified Barthel index, Instrumental ADL, NIHSS doi: $10.1017 /$ cjn.2020.149

Can J Neurol Sci. 2021; 48: 94-104

\section{INTRODUCTION}

In recent years, there has been an increase in the prevalence of stroke in China. A nationwide population-based survey reported that stroke is becoming a major cause of adult disability and premature death in China. ${ }^{1}$ Specifically, there has been a steady increase in ischemic stroke (IS) cases, which accounted

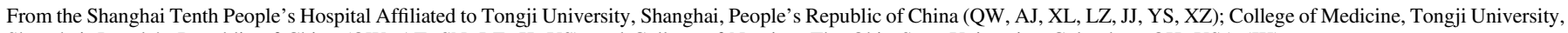
Shanghai, People's Republic of China (QW, AT, SN, LZ, JJ, YS); and College of Nursing, The Ohio State University, Columbus, OH, USA (JK)

Received February 14, 2020. Final Revisions Submitted July 4, 2020. Date of Acceptance July 6, 2020.

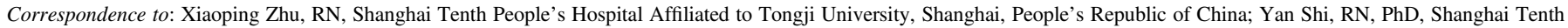
People's Hospital Affiliated to Tongji University, Shanghai, People's Republic of China. Email: 392470352@ qq.com Phone: 18917683111 
for $87.5 \%$ of all stroke cases from 2013 to $2015 .^{2}$ Early disability severity is an important predictor of functional outcome and the effectiveness of stroke treatment. ${ }^{3}$ Moreover, functional independence of stroke survivors ultimately affects their early rehabilitation, the ability to fully integrate into social life, and the resource cost. ${ }^{4,5}$

Previous studies have suggested that patients admitted to an acute stroke unit are inactive and physically limited during the first 2 weeks after stroke. ${ }^{6}$ Early evaluation of disability allows to prompt intervention and therapy, which provide a basis for early recovery planning and lead to better outcomes ultimately. Moreover, assessing the activities of daily living could assist nurses and caregivers to deliver proper care, health services, and living assistance. Ovbiagele et al. ${ }^{7}$ reported that the assessment of disability or dependence in activities of daily living could strongly predict long-term disability in patients with stroke, especially those who had ischemic stroke onset within 1 week. Given the availability of data in the hospital and the lack of long-term evaluation due to loss to follow-up, 1 week is considered as the best and most practical time point for measuring the direct effect of thrombolysis. ${ }^{8}$

Several instruments are available for assessing physical disability and dependency in patients with stroke. Among these instruments, the Modified Barthel Index (MBI), ${ }^{9}$ modified Rankin scale (mRs), ${ }^{10}$ and instrumental activities of daily living $\left(\right.$ IADL) ${ }^{11}$ are the most widely and frequently used in clinical practice and trials. Each instrument has its advantages and disadvantages. For example, the MBI assesses patients' basic activities; however, it lacks information on the IADL. Compared with the MBI, the mRs is considered as a global but more subjective scale. The IADL is related to cognitive changes, because it not only measures the ability of patients to live independently and take care of themselves, but also assesses cognitive, intellectual, and social skills by evaluating their ability to use the phone, manage finance, and take transportation. ${ }^{12}$ It is not usually or conveniently used among acute stroke survivors for completing the test content, which is difficult. Most previous studies assessed disability in stroke survivors during the rehabilitation and recovery period. ${ }^{13}$ For example, a previous qualitative review recommended the $\mathrm{MBI}$ and $\mathrm{mRs}$ as prognostic assessment tools for long-term outcomes after stroke. ${ }^{14}$ Cioncoloni et al. ${ }^{15}$ reported that $\mathrm{mRs}$ had better sensitivity in determining functional recovery at 6 months after stroke onset. However, few studies used those three instruments simultaneously on the assessment of physical activity in acute stroke survivors. Moreover, it remains unclear that which tool is more sensitive and more suitable to be used as a reference tool for acute stroke survivors.

In this study, we compared MBI, IADL, and mRs in stroke survivors in an acute care unit before post-stroke day 30. In the first stage, we administered four instruments for all patients during post-stroke days 5-7, including $\mathrm{mRs}$, MBI, IADL, and NIHSS assessments. In the second stage, we evaluated the NIHSS scores on the 30th day after post-stroke. We aimed to describe the distribution of the scores of the three instruments and factors affecting the recovery of prognosis on the 30th day after stroke by using NIHSS. Moreover, we aimed to compare the three instruments and determine the best and most sensitive measures for determining physical disability.

\section{Patients and Methods \\ Patients}

We conducted this prospective observational study at Shanghai Tenth People's Hospital Affiliated to Tongji University from June 2018 to November 2019. The inclusion criteria were a diagnosis of acute ischemic stroke and an age of $>18$ years. We excluded patients with dementia; no significant functional improvement after 48 hours of treatment; psychiatric history; and severe complications, including heart, renal, and hepatic failure, as well as patients that were isolated due to infectious diseases.

\section{Measures}

\section{Sociodemographic Questionnaire}

This questionnaire items included age, education, marital status, body mass index (BMI), hospitalization cost, length of hospital stay, career, and habits (sedentary lifestyle, smoking, and drinking habits).

\section{Clinical Characteristics}

We assessed the following clinical characteristics: infarct site, stroke severity, thrombolysis process, sleep situation as assessed by the Pittsburgh sleep quality index (PSQI), history of chronic disease (hypertension, coronary heart disease, atrial fibrillation, diabetes, and hyperlipidemia), and history of TIA. We obtained patients' clinical characteristics from the medical records. Based on the neuroimaging and clinical characteristics, experienced stroke neurologists made a definitive diagnosis of ischemic stroke. Moreover, neurologists and radiologists confirmed the stroke type and localization using head magnetic resonance imaging and/or computed tomography.

\section{Physical Disability Assessment}

In this study, we used the mRs, MBI, and IADL to assess physical disability.

The Modified Barthel Index (MBI). The MBI is a 10-item scale used to investigate the patient's capacity in activities of daily living (feeding, bowel control, bladder control, personal hygiene, transfer, dressing, ambulation, bathing, and stair climbing). ${ }^{15}$ The 10 subscale scores add up to a maximum possible score of 100 (independent) and minimum possible score of 0 (completely dependent). Moreover, a score of 0-40, 41-60, and 61-95 points indicates severe, moderate, and mild functional impairment. The Chinese version of the MBI has been reported to have good validity and reliability among patients with stroke. ${ }^{16}$

Modified Rankin Scale (mRs). The mRs is a 6-point scale: a 0 score indicates no symptoms at all; a 1 score indicates no significant disability despite symptoms and able to perform all usual duties; a 2 score indicates slight disability, and inability to perform all previous activities, but able to look after their own affairs without assistance; a 3 score indicates moderate disability, requiring some help but able to walk without assistance; a 4 score indicates moderately severe disability, and unable to 
walk and attend to their own bodily needs without assistance; and a 5 score indicates severe disability, bedridden, incontinent, and requiring constant nursing care and attention. ${ }^{17}$ This scale has poor sensitivity to change; however, it has relatively good psychometric properties, especially with respect to inter-rater reliability. ${ }^{18}$

Instrumental Activities of Daily Living (IADL). The IADL scale is an 8-item care worker-rated instrument that assesses the ability to use a telephone, shop, prepare food, housekeep, do laundry, use transportation modes, responsibly take medications, and handle finances. The total score ranges from 0 (the lowest function) to 8 (the highest function). In the IADL, disability is defined as presenting impairment in one or two of the eight IADL scale items. ${ }^{19}$ The IADL scores are correlated with tasks that require sufficient capacity to make good decisions and those that require significant interaction with the environment. ${ }^{20}$

The National Institute of Health Stroke Scale (NIHSS). The NIHSS is a 15-item neurologic examination stroke scale that assesses consciousness, ocular movement, vision, coordination, speech and language, sensory function, upper and lower limb strength, facial muscle function, and visual hemi-neglect. ${ }^{21}$ It reflects both the degree of neurological damage and the mobility of the patient. A higher score indicates greater stroke severity. The cutoff of NIHSS categories is 0-5 for mild stroke, 6-14 for moderate stroke, 15-24 for severe stroke, and $>24$ indicates a very severe stroke. ${ }^{22}$ NIHSS $>5$ includes all non-mild stroke categories. It is a well-validated assessment tool that is commonly used in acute-clinical stroke practice up to 3 months after stroke; moreover, it has shown good agreement with other outcome measures. ${ }^{23}$ Notably, the baseline scores have strong predictive validity that patient with a baseline score of $<5$ is considered to satisfy the discharge criteria. In this study, we considered an NIHSS score of $<5$ as a good outcome. ${ }^{22}$

\section{Sleep Assessment}

The Pittsburgh Sleep Quality Index (PSQI). The PSQI was proposed by Buyess et al. ${ }^{24}$ in 1989 and consists of 24 items. All items are divided into seven dimensions: subjective sleep quality, time to sleep, sleep time, sleep efficiency, sleep disorders, hypnotic drugs, and daytime function. Higher PSQI scores indicate more severe insomnia. Because it is easy to use and has good reliability and validity, it has become a commonly used scale for clinical assessment in psychiatry. ${ }^{25}$

\section{Procedure}

Three nurses were trained to use the three instruments, simultaneously; their consistency was assessed on 20 patients prior to study onset. We collected information on the sociodemographic and clinical characteristics from the medical records. The trained nurses conducted assessments for physical disability and the sleep situation. When a patient could not complete an interview, we used a proxy, for example, the nurse-in-charge or caregiver. We assessed the NIHSS score ${ }^{26}$ at 5-7 post-stroke days and at 30 post-stroke days. This open descriptive study received approval from the Shanghai Tenth People's Hospital Ethics Committee (SHSY-IEC-KY-4.0/17-47/01).

\section{Statistical Analysis}

We analyzed all data using the Statistical Package for Social Sciences version 21.0 software package (IBM Inc., Armonk, NY). For descriptive statistics, we used frequency and proportion for categorical variables and mean \pm standard deviation (SD) and number (percentage) for continuous variables.

We assessed the relationship among the MBI, IADL, and mRs scores in the acute stroke survivors. We used boxplots to present the distribution of MBI and IADL scores within each mRs grade. We used cross tabulation in Pearson's chi-squared test to determine the overlap of samples at each level of the three scales and Spearman's rank-order correlations test to analyze the correlations among the MBI, mRs, IADL, and the NIHSS (5-7 days) scores.

We used the Mann-Whitney U test for univariate analysis. We used a binary logistic regression model to assess the association between the NIHSS (30 days) scores and patient-related variables, including demographic and clinical characteristics. The NIHSS scores were dichotomized and coded as $\leq 5$ (good outcome) and $>5$ (bad outcome). The categorical variables were MBI (cutoff values: 40 and 60), IADL (cutoff values: 0 and 1), mRs (cutoff value: 2), and the NIHSS scores. We performed a multivariate analysis using variables with a p-value of $<0.05$ in the univariate analysis. Receiver operating characteristic (ROC) curves were used to assess the predictive value of the multivariate regression models. ROC analysis was conducted to examine the best assessment tool to predict the presence of NIHSS $>5$ on 5-7 days after stroke onset. We used the area under the curve (AUC) to assess the fit of the models. The results were interpreted as follows: $0.7-0.9$ as moderate accuracy and $0.5-0.7$ as low accuracy. We considered variables with a p-value of $<0.05$ as significant for all analyses.

\section{ReSUltS \\ Patient Characteristics}

We enrolled 136 acute stroke survivors. Table 1 summarizes the demographic and clinical characteristics.

\section{Distribution of the MBI and IADL Scores Related to the mRs Grades}

We determined the frequency distribution of the MBI and IADL scores within each $\mathrm{mRs}$ grade. There was a significant difference $(\mathrm{P}<0.001)$ in the distribution of the MBI and IADL median scores within the mRs grades (Kruskal-Wallis: 109.549 and 54.547 for MBI and IADL, respectively) (Figure 1).

Table 2 presents the number of patients overlapping at each level of the three scales. We observed that when the mRs score was $<2$, the MBI score was $>60$. With an increase in the $\mathrm{mRs}$ score, there was an increase in MBI scores that were $<60$. When the mRs score was equal to 5 , all the MBI scores were $<60$. The distribution of the IADL scores within the mRs grades overlapped when the $\mathrm{mRs}$ score was $<3$ points and $>4$ points. Given the meticulous classification of the IADL and its different emphasis compared with the mRs, the intersection of the intermediate scores is more complicated compared with that of MBI.

\section{Correlation among the Three Scales}

Spearman's correlation analysis revealed correlations among the three scales $(\mathrm{P}<0.001)$; specifically, the IADL, $\mathrm{mRs}$, and 
Table 1: Demographic and clinical characteristics of the participants

\begin{tabular}{|c|c|c|c|}
\hline Demographic Data & $\mathbf{n}(\%)$ & Clinical Data & $\mathbf{n}(\%)$ \\
\hline Gender & & The infarct region & \\
\hline Male & $94(69.1)$ & Vertebrobasilar region & 57(41.9) \\
\hline Female & $42(30.9)$ & $\begin{array}{c}\text { Bilateral cerebral } \\
\text { hemisphere }\end{array}$ & $9(6.6)$ \\
\hline Age & & $\begin{array}{r}\text { Right cerebral } \\
\text { hemisphere }\end{array}$ & $25(18.4)$ \\
\hline$\leq 40$ & $4(2.9)$ & $\begin{array}{l}\text { Left cerebral } \\
\text { hemisphere }\end{array}$ & 25(18.4) \\
\hline $40-50$ & $3(2.2)$ & Else & $20(14.7)$ \\
\hline $50-60$ & $34(25.0)$ & The use of thrombolysis & \\
\hline$>60$ & $95(69.9)$ & Yes & $21(15.4)$ \\
\hline BMI & & No & $115(84.6)$ \\
\hline$\leq 18.5$ & $2(17.6)$ & Sedentary & \\
\hline $18.5-24$ & $56(70.6)$ & Yes & 135(99.3) \\
\hline$>24$ & $78(8.8)$ & No & $1(0.7)$ \\
\hline Marriage & & Smoking & \\
\hline Unmarried & $15(11.0)$ & Yes & $63(46.3)$ \\
\hline Married & $121(89.0)$ & No & $73(53.7)$ \\
\hline Career & & Drinking & \\
\hline Incumbent & $11(8.1)$ & Yes & $37(27.2)$ \\
\hline Retirement & 125(91.9) & No & 99(72.8) \\
\hline Education & & Hypertension & \\
\hline Elementary and below & $10(7.3)$ & Yes & $82(60.3)$ \\
\hline Junior high school & $64(47.1)$ & No & $54(39.7)$ \\
\hline High school & $31(22.8)$ & Diabetes & \\
\hline Specialist and above & $31(22.8)$ & Yes & $31(22.8)$ \\
\hline Hospital costs (yuan) & & No & $105(77.2)$ \\
\hline $540-5840$ & $6(4.4)$ & Coronary heart disease & \\
\hline $5840-11,140$ & $86(63.2)$ & Yes & 15(11.0) \\
\hline $11,140-16,440$ & $36(26.5)$ & No & $121(19.0)$ \\
\hline $16,440-21,740$ & $4(2.9)$ & Atrial fibrillation & \\
\hline $21,740-52,803$ & $4(2.9)$ & Yes & $9(6.6)$ \\
\hline Stay at hospital & & No & 127(93.4) \\
\hline$\leq 7$ days & $38(27.9)$ & Hyperlipidemia & \\
\hline$>7$ days & $98(73.1)$ & Yes & $8(5.9)$ \\
\hline $\begin{array}{l}\text { Time from onset to } \\
\text { admission }\end{array}$ & & No & $128(94.1)$ \\
\hline$\leq 24$ hours & $82(60.3)$ & $\begin{array}{l}\text { The history of Transient } \\
\text { ischemic attack }\end{array}$ & \\
\hline $1-7$ days & $32(23.5)$ & Yes & 132(97.1) \\
\hline \multirow[t]{7}{*}{$>7$ days } & $22(16.2)$ & No & $4(2.9)$ \\
\hline & & PSQI scores & \\
\hline & & $\leq 7$ & $98(72.1)$ \\
\hline & & $>7$ & $38(27.9)$ \\
\hline & & mRs & \\
\hline & & $\leq 2$ scores & $38(27.9)$ \\
\hline & & $>2$ scores & $98(72.1)$ \\
\hline
\end{tabular}

Table 1. (Continued)

\begin{tabular}{l|l|l|c}
\hline Demographic Data & $\mathbf{n}(\boldsymbol{\%})$ & \multicolumn{1}{|c|}{ Clinical Data } & $\mathbf{n}(\%)$ \\
\hline & & NIHSS(30 days) & \\
\hline & & $\leq 5$ & $74(54.4)$ \\
\hline & & $>5$ & $62(45.6)$ \\
\hline & & MBI & \\
\hline & & $0-40$ scores & $16(11.8)$ \\
\hline & & $41-60$ scores & $45(33.1)$ \\
\hline & & $61-100$ scores & $75(55.1)$ \\
\hline & & IADL & \\
\hline & & 0 scores & $15(11.0)$ \\
\hline & & $1-7$ scores & $121(89.0)$ \\
\hline
\end{tabular}

$\mathrm{PSQI}=$ Pittsburgh sleep quality index; $\mathrm{IADL}=$ instrumental activities of daily living; $\mathrm{mRs}=$ modified rankin scale; $\mathrm{MBI}=$ modified barthel index; $\mathrm{BMI}=$ body mass index; NIHSS $=$ national institute of health stroke scale.
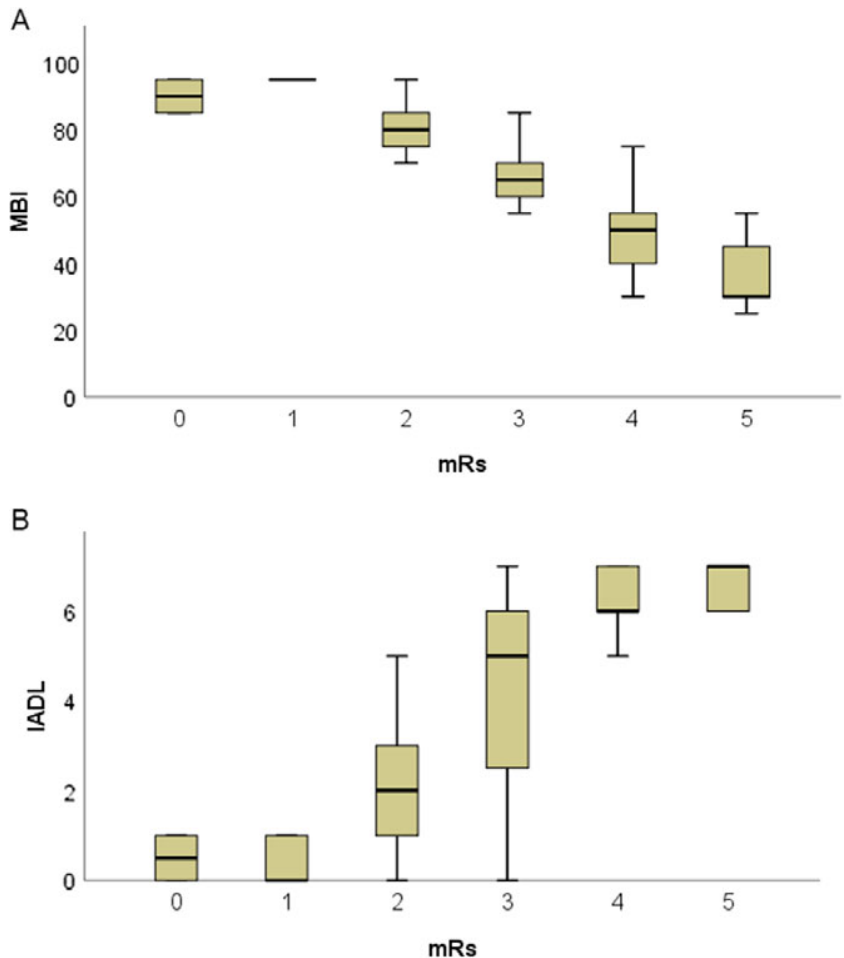

Figure 1: (A) Distribution of the MBI scores within the mRs grades. The frequency distribution of the MBI scores within each $m R s$ grade at 5-7 days is shown. The figure presents boxplots of the MBI score within each mRS grade at 5-7 days post-stroke. There is no overlap in the median frequency distribution at 5-7 days. There are significant differences between grade 5 and the remaining grades. (B) Distribution of the IADL scores within the $m R s$ grades. The frequency distribution of the IADL scores within each $m R s$ level at 5-7 days is shown. The figure shows the boxplots of the IADL score within each $m R S$ grade at 5-7 days post-stroke. There is no overlap in the median frequency distribution at 5-7 days. There are significant differences between grade 5 and the remaining grades.

MBI were negatively correlated $(r<0)$, while the IADL and mRs were positively correlated $(r>0)$. Moreover, there was a high correlation between the $\mathrm{mRs}$ and MBI $(\mathrm{r}=-0.870)$ and a 
Table 2: Overlapping of samples at each level of the three scales

\begin{tabular}{|c|c|c|c|c|c|c|c|c|c|c|c|c|c|c|}
\hline \multirow{2}{*}{$\begin{array}{l}\text { Instrument } \\
\text { scores }\end{array}$} & & \multicolumn{3}{|c|}{ MBI } & \multirow[t]{2}{*}{ Total } & \multicolumn{7}{|c|}{ IADL score } & \multicolumn{2}{|c|}{ Total } \\
\hline & & 0 & 1 & 2 & & 0 & 1 & 2 & 3 & 4 & 5 & 6 & 7 & \\
\hline & 0 & 0 & 0 & 0 & 0 & 1 & 1 & 0 & 0 & 0 & 0 & 0 & 0 & 2 \\
\hline \multirow[t]{5}{*}{ mRs score } & 1 & 5 & 0 & 0 & 5 & 3 & 2 & 0 & 0 & 0 & 0 & 0 & 0 & 5 \\
\hline & 2 & 32 & 0 & 0 & 32 & 5 & 6 & 8 & 6 & 3 & 4 & 0 & 0 & 32 \\
\hline & 3 & 32 & 12 & 0 & 44 & 3 & 4 & 4 & 2 & 6 & 9 & 13 & 3 & 44 \\
\hline & 4 & 4 & 31 & 12 & 47 & 3 & 2 & 2 & 1 & 2 & 2 & 17 & 18 & 47 \\
\hline & 5 & 0 & 2 & 4 & 6 & 0 & 0 & 0 & 0 & 0 & 0 & 2 & 4 & 6 \\
\hline Total & & 75 & 45 & 16 & 136 & 15 & 15 & 14 & 9 & 11 & 15 & 32 & 25 & 136 \\
\hline
\end{tabular}

MBI = modified barthel index.

Note: $0=61-99$ scores; $1=41-60$ scores; $2=0-40$ scores in the MBI.

Table 3: The correlation between the three scales

\begin{tabular}{l|c|c|c|c|c|c}
\hline & \multicolumn{2}{|c|}{ mRs scores } & \multicolumn{2}{c}{ MBI scores } & \multicolumn{2}{c}{ IADL scores } \\
\hline & $\boldsymbol{r}$ & $\boldsymbol{P}$ & $\boldsymbol{r}$ & $\boldsymbol{p}$ & $\boldsymbol{r}$ \\
\hline IADL scores & 0.559 & $<0.001$ & -0.587 & $<0.001$ & & \\
\hline MBI scores & -0.870 & $<0.001$ & & & & \\
\hline
\end{tabular}

IADL $=$ instrumental activities of daily living; $\mathrm{mRs}=$ modified rankin scale; $\mathrm{MBI}=$ modified barthel index.

Table 4: The correlation between the three scales and the NIHSS (admission)

\begin{tabular}{l|c|c|c|c|c|c}
\hline & \multicolumn{2}{|c|}{ mRs scores } & \multicolumn{2}{c}{ MBI scores } & \multicolumn{2}{c}{ IADL scores } \\
\hline & $\boldsymbol{r}$ & $\boldsymbol{p}$ & $\boldsymbol{r}$ & $\boldsymbol{p}$ & $\boldsymbol{r}$ \\
\hline NIHSS scores & 0.769 & $<0.001$ & -0.823 & $<0.001$ & 0.569 & $<0.001$ \\
\hline
\end{tabular}

$\mathrm{IADL}=$ instrumental activities of daily living; $\mathrm{mRs}=$ modified rankin scale; $\mathrm{MBI}=$ modified barthel index.

moderate correlation of the IADL and $\mathrm{mRs}$ with MBI $(\mathrm{r}=0.559$ and -0.587 , respectively) (Table 3 ).

\section{Correlation between Each of the Three Scales and the NIHSS (5-7 Days)}

Spearman correlation analysis revealed correlations between each of the three scales and the NIHSS (days 5-7) $(\mathrm{P}<0.001)$. Specifically, there was a negative correlation of the NIHSS (days 5-7) with the MBI $(r<0)$ and a positive correlation of the NIHSS (admission) with the IADL and $\mathrm{mRs}(\mathrm{r}>0)$. There was a high correlation of the MBI and mRs with NIHSS (days 5-7) ( $\mathrm{r}=-0.823$ and 0.769 , respectively) and a moderate correlation of the IADL with NIHSS (days 5-7) $(r=0.569)$ (Table 4).

\section{Univariate and Multivariate Analysis of the NIHSS Scores (Day 30)}

Table 5 presents the results of univariate and multivariate analyses of the NIHSS scores (day 30). Thrombolysis $(\mathrm{P}<0.001)$ and time from onset to admission $(\mathrm{P}=0.016)$ showed statistically significant associations with the NIHSS score (day 30). Binary logistic regression analysis revealed the significant association between thrombolysis and the NIHSS score (day 30) after adjusting for the variable time from onset to admission. Concomitantly, thrombolysis was an independent protective factor of NIHSS (day 30) $(\mathrm{OR}=0.057,95 \% \mathrm{CI}=0.042-0.585$, $\mathrm{P}=0.006)$.

Receiver Operator Curves of the Predictive Ability of the MBI, IADL, and mRs Compared to that of the NIHSS (Day 30)

ROC curves showed the predictive power of the multivariable model for the three scales (Figure 2). Figure 2 demonstrates that the MBI score (cutoff value: 60), IADL score (cutoff value: 1), and mRs scores (cutoff value: 2 ) had the best sensitivity, which indicates that these scales had the best prognosis for acute stroke. The area under the ROC curve for the model for the mRs score (cutoff value: 2), MBI scores (cutoff value: 60), and IADL score (cutoff value: 1) was 0.831 (95\% CI: 0.761-0.901), 0.749 (95\% CI: 0.664-0.835), and 0.698 (95\% CI: 0.608-0.789). 
Table 5: Univariate and multivariate analysis of factors associated with the NIHSS (day 30)

\begin{tabular}{|c|c|c|c|c|c|c|c|c|}
\hline \multirow{3}{*}{$\begin{array}{l}\text { Factors } \\
\text { Gender }\end{array}$} & \multirow{3}{*}{ n (\%) } & \multirow{2}{*}{\multicolumn{2}{|c|}{$\begin{array}{c}\text { Univariate analysis* } \\
\text { NIHSS ( } 5 \text { cutoff) } \\
\text { Statistics } P \\
\end{array}$}} & \multirow{2}{*}{$\begin{array}{c}\text { Multivariate analysis } \\
\text { NIHSS(5 cutoff) OR } P \\
95 \% \text { CI }\end{array}$} & \multirow{3}{*}{ Factors } & \multirow{3}{*}{ n $(\%)$} & \multirow{2}{*}{\multicolumn{2}{|c|}{$\begin{array}{c}\text { Univariate analysis } \\
\text { NIHSS ( } 5 \text { cutoff) } \\
\text { Statistics } P \\
\end{array}$}} \\
\hline & & & & & & & & \\
\hline & & & & & & & & \\
\hline Male & $94(69.1)$ & 0.437 & 0.491 & & Incumbent & $11(8.1)$ & $<0.001$ & 0.993 \\
\hline Female & $42(30.9)$ & & & & Retirement & $125(91.9)$ & & \\
\hline Age & & & & & Coronary heart disease & & & \\
\hline$\leq 40$ & $4(2.9)$ & 2.052 & 0.152 & & Yes & $15(11.0)$ & 0.405 & 0.525 \\
\hline $40-50$ & $3(2.2)$ & & & & No & $121(19.0)$ & & \\
\hline $50-60$ & $34(25.0)$ & & & & Sedentary & & & \\
\hline$>60$ & $95(69.9)$ & & & & Yes & $135(99.3)$ & 0.838 & 0.360 \\
\hline BMI & & & & & No & $1(0.7)$ & & \\
\hline$\leq 18.5$ & $2(17.6)$ & 0.010 & 0.940 & & Smoking & & & \\
\hline $18.5-24$ & $56(70.6)$ & & & & Yes & $63(46.3)$ & 0.009 & 0.923 \\
\hline$>24$ & $78(8.8)$ & & & & No & 73(53.7) & & \\
\hline The history of TIA & & & & & Drinking & & & \\
\hline Yes & $4(2.9)$ & 0.699 & 0.403 & & Yes & $37(27.2)$ & 0.003 & 0.959 \\
\hline No & $132(97.1)$ & & & & No & $99(72.8)$ & & \\
\hline Atrial fibrillation & & & & & Hypertension & & & \\
\hline Yes & $9(6.6)$ & 1.783 & 0.191 & & Yes & $82(60.3)$ & 0.047 & 0.829 \\
\hline No & 127(93.4) & & & & No & $54(39.7)$ & & \\
\hline Hyperlipidemia & & & & & Diabetes & & & \\
\hline Yes & $8(5.9)$ & 0.223 & 0.637 & & Yes & $31(22.8)$ & 1.641 & 0.200 \\
\hline No & $128(94.1)$ & & & & No & $105(77.2)$ & & \\
\hline The use of thrombolysis & & & & & Region of infarct & & & \\
\hline \multirow[t]{2}{*}{ Yes } & \multirow[t]{2}{*}{$21(15.4)$} & \multirow[t]{2}{*}{12.195} & \multirow[t]{2}{*}{$<0.001 \dagger$} & $0.057 \ddagger 0.006$ & \multirow[t]{2}{*}{ Vertebrobasilar region } & \multirow[t]{2}{*}{$57(41.9)$} & \multirow[t]{2}{*}{0.265} & \multirow[t]{2}{*}{0.492} \\
\hline & & & & $(0.042-0.585)$ & & & & \\
\hline No & $115(84.6)$ & & & & $\begin{array}{c}\text { Bilateral cerebral } \\
\text { hemisphere }\end{array}$ & $9(6.6)$ & & \\
\hline $\begin{array}{l}\text { Time from onset to } \\
\text { admission }\end{array}$ & & & & & $\begin{array}{r}\text { Right cerebral } \\
\text { hemisphere }\end{array}$ & $25(18.4)$ & & \\
\hline \multirow[t]{2}{*}{$\leq 24$ hours } & \multirow[t]{2}{*}{$82(60.3)$} & \multirow[t]{2}{*}{6.103} & \multirow[t]{2}{*}{$0.016^{\dagger}$} & 1.4950 .112 & \multirow[t]{2}{*}{ Left cerebral hemisphere } & \multirow[t]{2}{*}{$25(18.4)$} & & \\
\hline & & & & $(0.910-2.454)$ & & & & \\
\hline $1-7$ days & $32(23.5)$ & & & & Else & $20(14.7)$ & & \\
\hline$>7$ days & $22(16.2)$ & & & & Marriage & & & \\
\hline Education & & & & & Unmarried & $15(11.0)$ & 0.345 & 0.538 \\
\hline Elementary and below & $10(7.3)$ & 0.005 & 0.646 & & Married & $121(89.0)$ & & \\
\hline Junior high school & $64(47.1)$ & & & & PSQI score & & & \\
\hline High school & $31(22.8)$ & & & & $\leq 7$ & $98(72.1)$ & 3.196 & 0.074 \\
\hline Specialist and above & $31(22.8)$ & & & & $>7$ & $38(27.9)$ & & \\
\hline
\end{tabular}

$\mathrm{BMI}=$ body mass index; TIA = transient ischemic attack; PSQI = Pittsburgh sleep quality index.

*Mann-Whitney U test.

$\dagger$ Statistically significant $(\mathrm{P}<0.05)$.

†Odds ratio indicated that patients with functional recovery (NIHSS $<5$ ) were 0.057 times more likely to be functionally impaired (NIHSS $>5$ ) at 30 days compared to patients who did not undergo thrombolysis. 


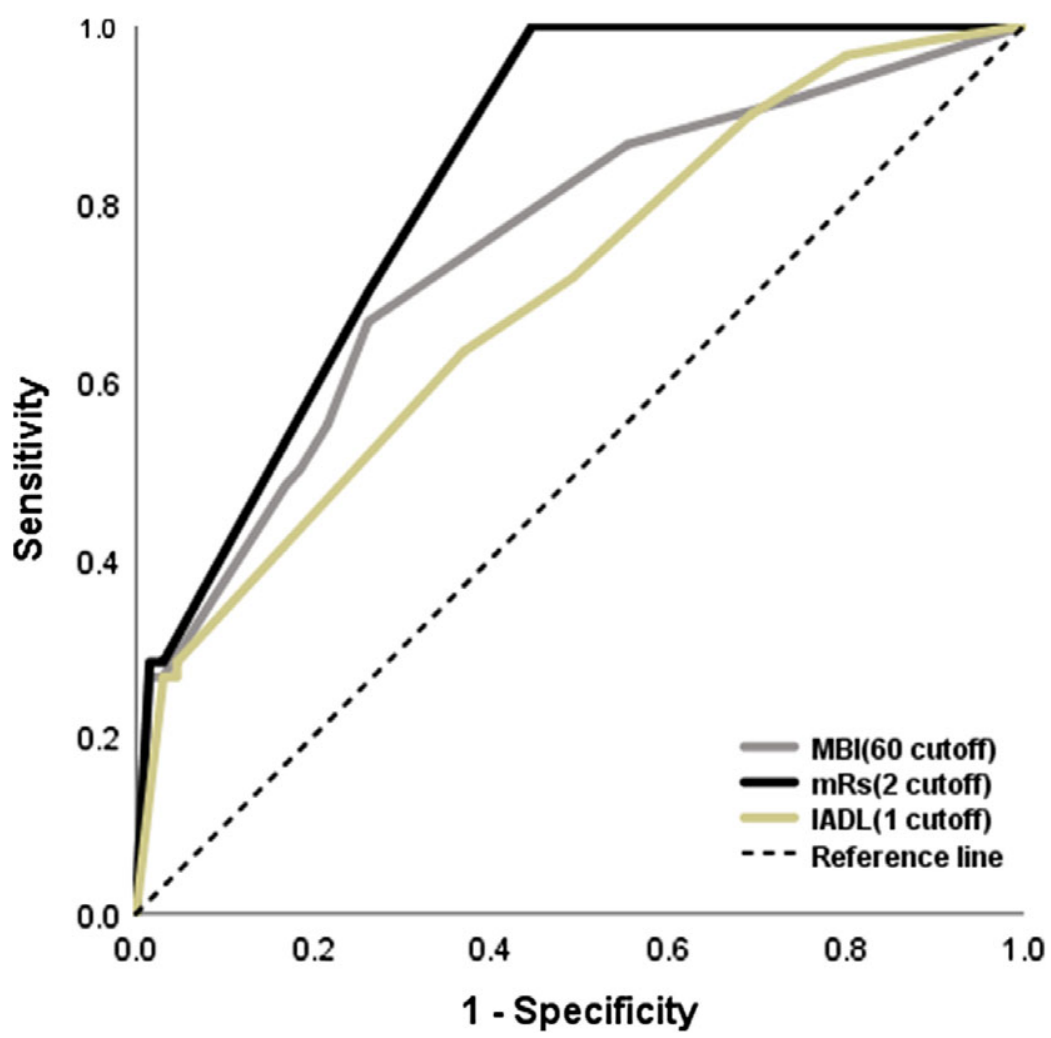

Figure 2: The area under the ROC curve shows an improved predictive value $(0.698$ 0.831 ) for total prognosis after inclusion of the MBI score (cutoff value: 60), IADL score (cutoff value: 1), and mRs score (cutoff value: 2).

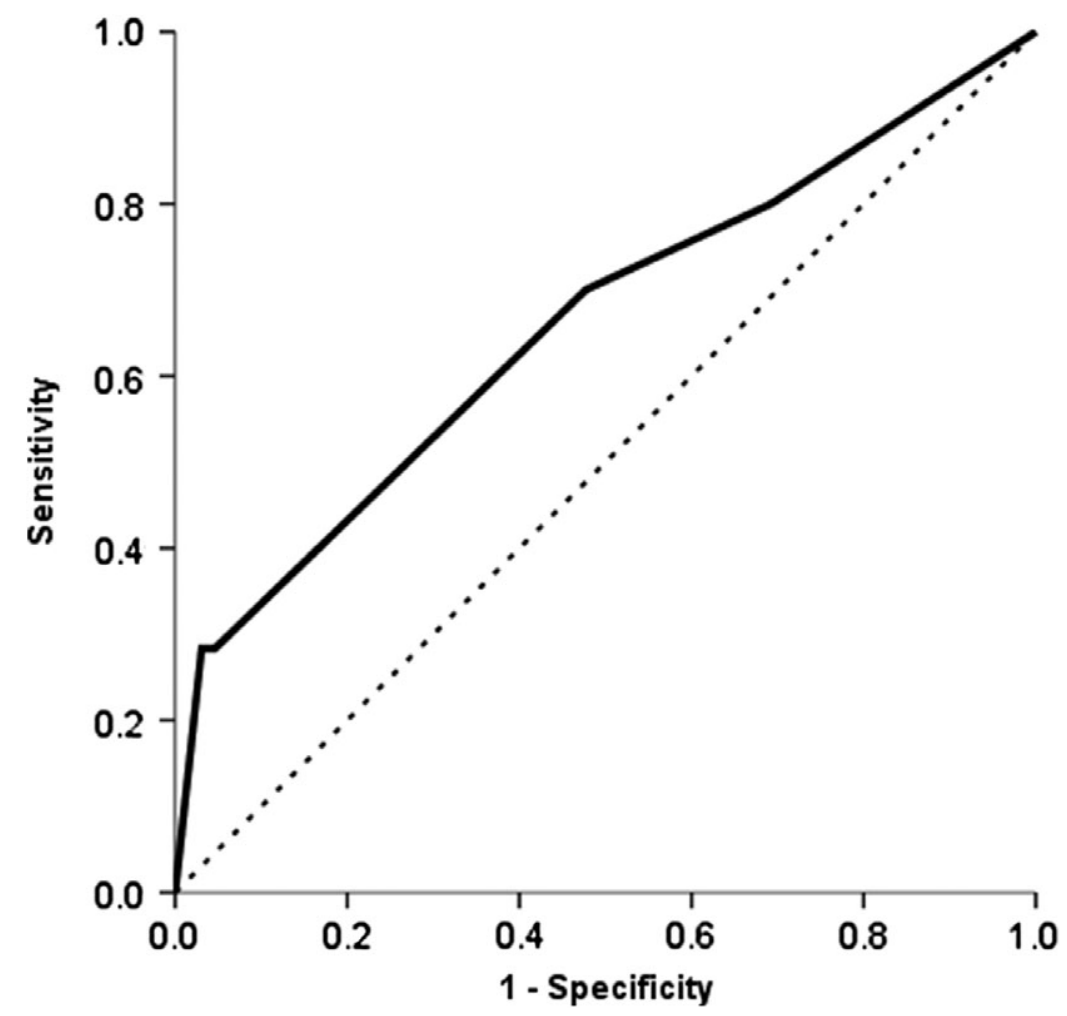

Figure 3: The area under the ROC curve of the multivariable model for the NIHSS (day 30 ) is 0.659 (95\% CI: 0.563-0.755) with the inclusion of thrombolysis and time from onset to admission as independent variables. 


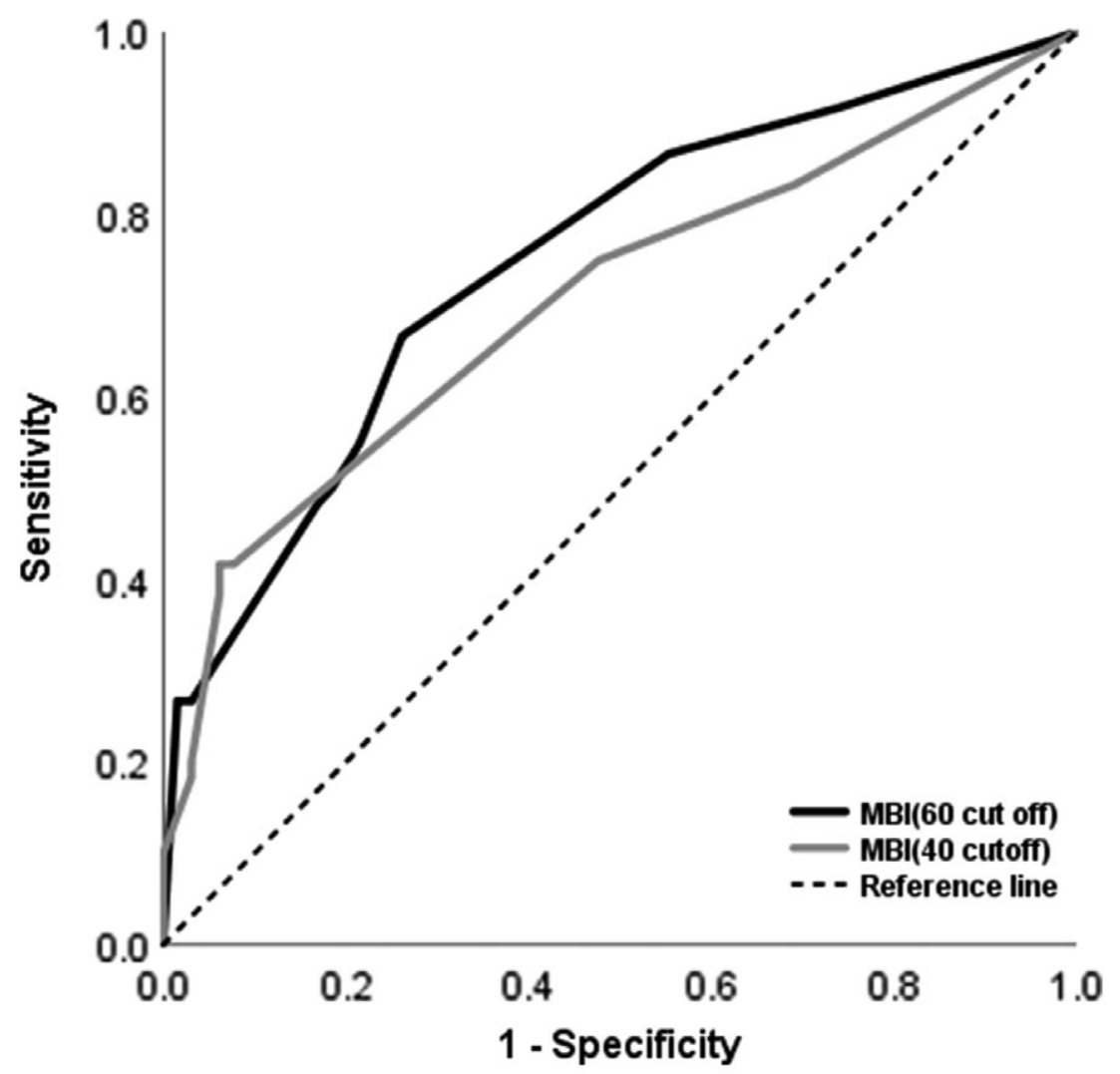

Figure 4: The area under the ROC curve of the multivariable model for the MBI (cutoff value: 40) is 0.706 (95\% CI: 0.613-0.798) with the inclusion of thrombolysis, time from onset to admission, and the MBI score (cut off value: 40) as independent variables. The area under the ROC curve of the multivariable model for the MBI (cutoff value: 60) is 0.749 (95\% CI: $0.664-0.835$ ) with the inclusion of thrombolysis, time from onset to admission, and MBI (cutoff value: 60) as independent variables.

Figure 3 shows the predictive power of the NIHSS (day 30) in the multivariable model. The area under the ROC curve for the model for the NIHSS (day 30) was 0.659 (95\% CI: 0.563-0.755). The regression analysis model that included thrombolysis and time from onset to admission as the independent variables showed low sensitivity for explaining stroke prognosis.

Figure 4 presents the predictive power of the multivariable model for the MBI (cutoff value: 60 and cutoff value: 40 , respectively). The area under the ROC curve for the model for the MBI (cutoff value: 40 ) and MBI (cutoff value: 60 ) was 0.706 (95\% CI: $0.613-0.798$ ) and 0.749 (95\% CI: 0.664-0.835), respectively. Both regression analysis models with the inclusion of thrombolysis and time from onset to admission as independent variables showed moderate sensitivity for explaining acute stroke prognosis.

Figure 5 presents the predictive power of the multivariable model for the IADL (using cutoff values of 0 or 1). The area under the ROC curve for two different models of the IADL (cutoff value: 0 ) and IADL (cutoff value: 1 ) was 0.665 (95\% CI: $0.570-0.760$ ) and 0.698 (95\% CI: 0.608-0.789). Both regression analysis models, with the inclusion of thrombolysis and time from onset to admission as independent variables, showed low sensitivity for explaining acute stroke prognosis.

\section{Discussion}

We aimed to compare three assessment tools of physical disability at two time points in the acute post-stroke stage (within 30 days after stroke) among first-stroke survivors. We observed a high correlation among the three assessment tools $(\mathrm{P}<0.05)$. Although they all measured physical disability, they placed emphasis on different activity aspects. The MBI and mRs measure the degree of assistance required from other people to perform basic activities of daily living, while the IADL places emphasis on assistance required from devices. This could have contributed to the significant differences $(\mathrm{P}<0.001)$ in the distribution of the MBI and IADL scores within the mRs grades (Figures 1 and 2). Our study is consistent with a previous work, ${ }^{15}$ which shows that the MBI score distribution within $\mathrm{mRS}$ grades overlapped at 10 days. Kwon et al. assessed the frequency distribution of MBI scores within mRs grades and showed that the highest MBI scores (95-100, indicating excellent to complete recovery) corresponded to mRs grades $0,1,2$, and $4 .^{27}$ The MBI is used to assess activity function; however, its efficiency is compromised when administered too early (within 5 days post-stroke). ${ }^{28}$

The American Heart Association/American Stroke Association recommends early assessment of patients with acute 


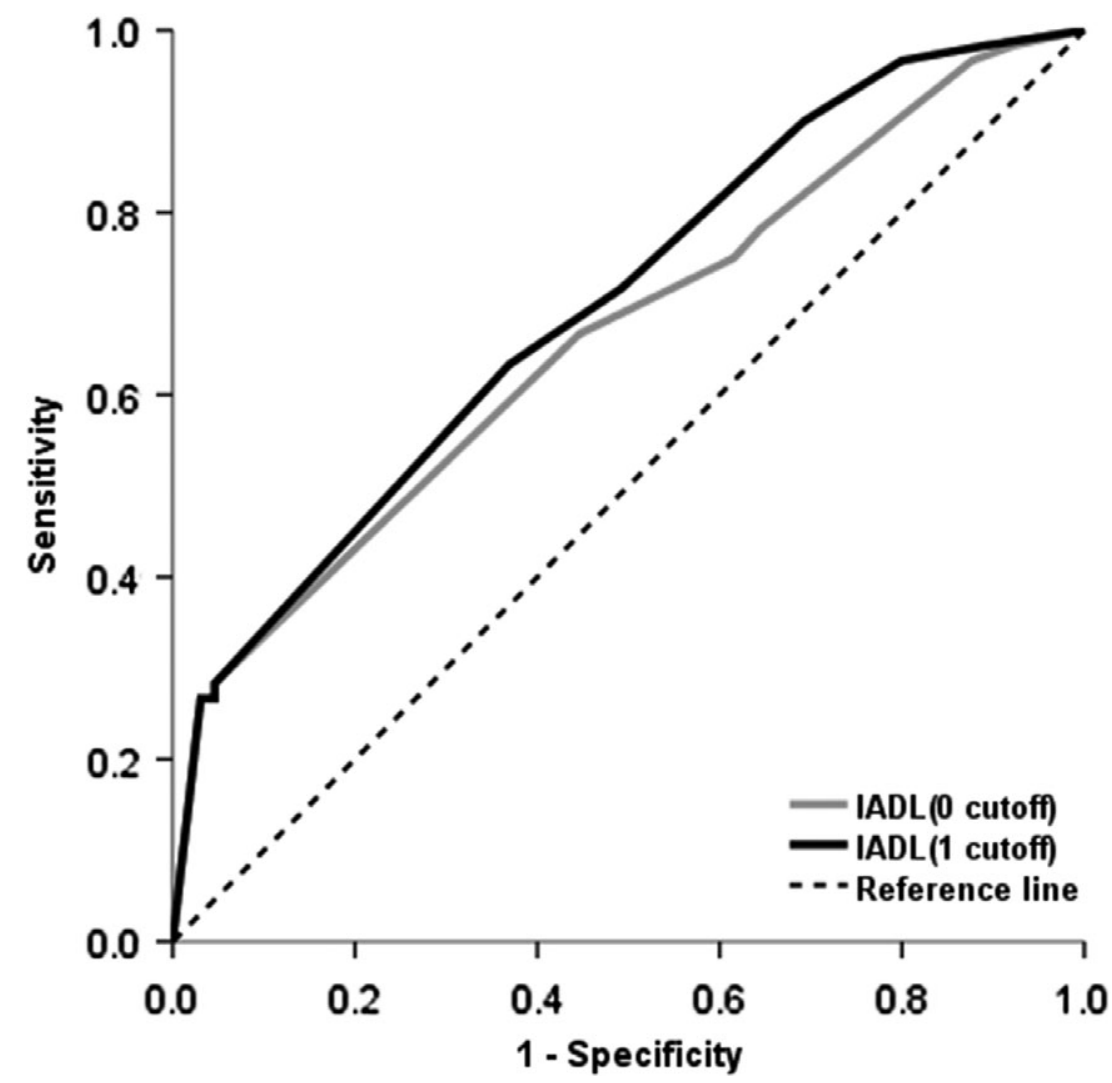

Figure 5: The area under the ROC curve of the multivariable model for the IADL (cutoff value: 0 ) is 0.665 (95\% CI: 0.570-0.760) with the inclusion of thrombolysis, time from onset to admission, and the IADL (cutoff value: 0 ) as independent variables. The area under the ROC curve of the multivariable model for the IADL (cutoff value: 1 ) is $0.698(95 \%$ CI: 0.608 0.789 ) with the inclusion of thrombolysis, time from onset to admission, and IADL (cutoff value: 1) as independent variables.

ischemic stroke, ${ }^{29}$ which could be facilitated by accurate assessment tools, which help to screen patients with the ability to perform activities and evaluate the effectiveness of rehabilitation treatment. The NIHSS is an important scale that reflects strokeinduced global neurological and motor impairment. Compared with the other two scales, we found that the MBI had the highest correlation $(\mathrm{r}=-0.823, \mathrm{P}<0.001)$ with the NIHSS. A previous study reported that the MBI is more reliable for disability assessment than the mRs. ${ }^{23}$ The inter-rater reliability and internal consistency of the MBI can be assessed, while the internal item consistency of the mRs cannot be evaluated, because it is onedimensional. Compared to the mRs, the MBI is considered less subjective since it allows for objective and specific evaluation, while the mRs obscurely describes the activity levels. As a disability measure, the mRs subsumes some items in the IADL and MBI (e.g., walking, dressing, bathing) and emphasizes compromised motor function. However, the mRs is more sensitive than the MBI in the measurement of stroke disability. ${ }^{30}$

It is recognized that activity ability could predict long-term outcomes and independence in the subsequent recovery period in patients with stroke. Univariate and multivariate analyses showed that thrombolysis (95\% CI: 0.042-0.585; P=0.006) was independently associated with the NIHSS (day 30). A previous study reported an association of thrombolysis with post-stroke outcome. ${ }^{31}$ Moreover, we compared the sensitivity and specificity of the three assessment tools in predicting stroke severity at the end of the acute stage. The multivariate analysis model with the $\mathrm{mRs}$ (cutoff value: 2) had the highest AUC (Figure 3), indicating that it could better predict functional recovery after acute stroke. Consistent with our findings, Dewilde et al. $^{4}$ reported that prognostic outcomes were associated with increasing levels of the mRs: in the first few months after a stroke, higher mRs scores were associated with higher disability levels and a higher cost of rehabilitation in the first month after the stroke. Moreover, the predictive validity of the mRs is indicated by the association between short-term and long-term post-stroke care needs. ${ }^{32}$ With respect to the IADL and MBI, different cutoff values showed different ROC curves and AUCs. Specifically, the AUCs of the MBI (cutoff value: 60) and the MBI (cutoff value: 40) were 0.794 (95\% CI: 0.664-0.835) and 0.706 (95\% CI: 0.613-0.798), respectively. On the other hand, the AUCs of the IADL (cutoff value: 0) and the IADL (cutoff value: 1) were 0.665 (95\% CI: $0.570-0.760)$ and 0.698 (95\% CI: 0.608-0.789), respectively. Therefore, when using the MBI or IADL to predict the short-term stroke severity, moderate scores of the MBI (cutoff value: 60) and IADL (cutoff value: 1) could be employed as predictive factors. 


\section{LIMITATIONS}

Our study has several limitations. First, we enrolled first ischemic stroke survivors during the acute stage (30 days after stroke). The main short-term outcome was the NIHSS score at day 30; however, other studies could employ mortality and assess readmission rates with larger samples. Additionally, in this study, we only assessed physical disability at a single time point rather than multiple time points throughout the 30 days. Finally, we did not assess and account for the relevant biochemical markers.

\section{Conclusions and Policy Implications in Practice}

In summary, compared with the other two instruments, we found that the BI was the best scale for reflecting disability in physical activity in acute stroke survivors; moreover, it was highly correlated with the NIHSS. This indicates that the MBI could assist nurses and caregivers to accurately assess physical disability and functional independence, which providing information for the degree of assistant and early as well as making a personal early rehabilitation plan in the acute stage. Furthermore, we found that the mRs showed better accuracy in the prediction of short-term stroke severity, which is consistent with previous findings regarding its utility for long-term outcomes. The level of mRs could guide doctors and nurses to make a proper decision for the follow-up plan. The findings are relevant to stroke care and might be meaningful for the rational utilization of medical resources.

\section{ACKNOWLedgments}

This research was funded by the National Natural Science Foundation of China (71704135).

\section{CONFLICT OF InTEREST}

None.

\section{Statement of Authorship}

QW, YS, and XPZ were responsible for the study's concept and design. AIT and SZN did the statistical analysis and data collection. QW wrote the paper. SZN, APJ, XQL, and LZ contributed to the analysis and interpretation of the data. JXJ and $\mathrm{JK}$ were responsible for subsequent drafts and revision.

\section{REFERENCES}

1. Wang W, Jiang B, Sun H, et al. Prevalence, incidence, and mortality of stroke in China. Circulation. 2017;135(8):759.

2. Tian D, Yang Q, Dong Q, Li N, Fan D. Trends in stroke subtypes and vascular risk factors in a stroke center in China over 10 years. Sci Rep. 2018;8(1):5037.

3. Banks JL, Marotta CA. Outcomes validity and reliability of the modified Rankin scale: implications for stroke clinical trials: a literature review and synthesis. Stroke. 2007;38(3):1091-6.

4. Dewilde S, Annemans L, Peeters A, et al. Modified Rankin scale as a determinant of direct medical costs after stroke. Int J Stroke. 2017;12:392-400.

5. Gialanella B, Santoro R, Ferlucci C. Predicting outcome after stroke: the role of basic activities of daily living. Eur J Phys Rehabil Med. 2013;49(5):629-37.

6. Bernhardt, J. Inactive and alone: physical activity within the first 14 days of acute stroke unit care. Stroke. 2004;35(4):1005-9.
7. Ovbiagele B, Saver JL. Day-90 acute ischemic stroke outcomes can be derived from early functional activity level. Cerebrovasc Dis. 2010;29(1):50-6.

8. Cappellari M, Moretto G, Bovi P. Day-7 modified Rankin scale score as the best measure of the thrombolysis direct effect on stroke? J Thromb Thrombolys. 2012;36(3):314-5.

9. Collin C, Wade DT, Davies S, Horne V. The Barthel ADL Index: a reliability study. Int Disabil Stud. 1988;10(2):61-3.

10. Bamford JM, Sandercock PA, Warlow CP, Slattery J. Interobserver agreement for the assessment of handicap in stroke patients. Stroke. 1989;20(6):828.

11. Gladman JRF. Measurement of instrumental activities of daily living in stroke. Stroke. 1995;26(11):2197.

12. Zucchella C, Federico A, Martini A, et al. Neuropsychological testing. Pract Neurol. 2018;18(3):227-37.

13. Burns SP, White BM, Magwood G, et al. Racial and ethnic disparities in stroke outcomes: a scoping review of post-stroke disability assessment tools. Disabil Rehabil. 2019;41(15):1835-45.

14. Huybrechts KF, Jaime Caro J. The Barthel Index and modified Rankin scale as prognostic tools for long-term outcomes after stroke: a qualitative review of the literature. Curr Med Res Opin. 2007;23(7):1627-36.

15. Cioncoloni D, Piu P, Tassi R, Acampa M, Mazzocchio R. Relationship between the modified Rankin scale and the Barthel Index in the process of functional recovery after stroke. Neurorehabilitation. 2012;30(4):315-22.

16. Leung SOC, Chan CCH, Shah S. Development of a Chinese version of the modified Barthel Index validity and reliability. Clin Rehabil. 2007;21(10):912-22.

17. Haan RD, Limburg M, Bossuyt P, Meulen JVD, Aaronson N. The clinical meaning of Rankin 'handicap' grades after stroke. Stroke. 1995;26(11):2027-30.

18. Fearon P, Mcarthur KS, Garrity K, Graham LJ, Quinn TJ. Prestroke modified Rankin stroke scale has moderate interobserver reliability and validity in an acute stroke setting. Stroke. 2012;43(12):3184-8.

19. Yang M, Xu H, Yang L, Jiang J, Dong B. Metabolic syndrome and disability in Chinese nonagenarians and centenarians. Aging Clin Exp Res. 2018;30(8):943-9.

20. Millán-Calenti JC, Tubío J, Pita-Fernández S, et al. Prevalence of functional disability in activities of daily living (ADL), instrumental activities of daily living (IADL) and associated factors, as predictors of morbidity and mortality. Arch Gerontol Geriatr. 50(3):306-10.

21. Lyden P, Lu M, Jackson C, et al. Underlying structure of the National Institutes of Health Stroke Scale: results of a factor analysis. Stroke. 1999;30(11):2347-54.

22. Martin TR, Alastair W, Jesse D, Terrence JQ. Functional assessment for acute stroke trials: properties, analysis, and application. Front Neurol. 2018;9:191.

23. Alvarez-Sabín J, Molina CA, Montaner J, Arenillas JF, Quintana M. Effects of admission hyperglycemia on stroke outcome in reperfused tissue plasminogen activator-treated patients. Stroke. 2003;34(5):1235-41.

24. Buysse DJ, Iii C F R, Monk T H, et al. The Pittsburgh sleep quality index: a new instrument for psychiatric practice and research. Psychiatry Res. 1989;28(2):193-213.

25. Zheng B, Li M, Wang KL, et al. Analysis of the reliability and validity of the Chinese version of Pittsburgh sleep quality index among medical college students. Beijing Da Xue Bao Yi Xue Ban. 2016;48(3):424-8.

26. Kwah LK, Diong J. National Institutes of Health Stroke Scale (NIHSS). J Physiother. 2014;60(1):61.

27. Kwon S, Hartzema AG, Duncan PW, Minlai S. Disability measures in stroke relationship among the Barthel Index, the functional independence measure, and the modified Rankin scale. Stroke. 2004;35(4):918-23.

28. Quinn TJ, Langhorne P, Stott DJ. Barthel Index for stroke trials: development, properties, and application. Stroke. 2011;42(4): 1146-51.

29. Winstein CJ, Stein J, Arena R, et al. Guidelines for adult stroke rehabilitation and recovery: a guideline for healthcare 
professionals from the American Heart Association/American Stroke Association. Stroke. 2016;47(6):e98we-169.

30. Balu S. Differences in psychometric properties, cut-off scores, and outcomes between the Barthel Index and modified Rankin scale in pharmacotherapy-based stroke trials: a systematic literature review. Curr Med Res Opin. 2009;25(6):1329-41.
31. Saňák D, Herzig R, Zapletalová J, et al. Predictors of good clinical outcome in acute stroke patients treated with intravenous thrombolysis. Acta Neurol Scand. 2011;123(5):339-44.

32. Mayo NE, Wood-Dauphinee S, Cote R, et al. There's no place like home : an evaluation of early supported discharge for stroke. Stroke. May 2000;31(5):1016-23. 